\title{
Damping of subsynchronous oscillations using adaptive controllers tuned by artificial neutral networks
}

Y.-Y. Hsu, PhD

L.-H. Jeng, MSc

Indexing terms: Subsynchronous resonance, Static VAR compensator, Power system stabiliser, Artificial neural network, Optimal control, Back propagation

Abstract: Artificial neural networks (ANNs) are utilised to adapt the controller gains of two widely used control schemes i.e. static VAR compensators (SVC) and excitation controllers (EC), for the damping of subsynchronous resonance (SSR) on a power system. To have good damping characteristics of SSR modes over a wide range of operating conditions, the parameters of the adaptive controllers are adapted based on generator loading conditions. Multilayer feedforward artificial neural networks (ANNs) are developed to serve for the purpose of controller parameter adaptation. The inputs to the ANN include the real power output $P$ and reactive power output $Q$ which characterise generator loading conditions. The outputs from the ANN are the desired controller gains. Time domain simulations are also performed on the IEEE first benchmark model to demonstrate the effectiveness of the proposed adaptive control schemes.

\section{List of symbols}

general

$A, B, C$

$Q, R, S$

$=$ system matrix

$=$ performance index of optimal control

$H(s), H_{l}(s), H_{\omega}(s)=$ transfer function of the compensator

$\theta, \omega=$ rotor angle and rotor speed

$\delta \quad=$ generator torque angle

$V, I=$ voltage and current

$R, X \quad=$ resistance and reactance

$B_{L}, B_{C} \quad=$ susceptances of the inductor and capacitor

$T \quad=$ torque

$E_{f d} \quad=$ per unit output voltage of exciter

$V_{R} \quad=$ voltage regulator output

$a \quad=$ actuator signal

$g \quad=$ governor opening

$e_{c} \quad=$ capacitor voltage

$M \quad=$ moment of inertia $(M=2 H)$

$K=$ spring constant

$L_{T} \quad=$ transformer inductance

(C) IEE, 1995

Paper 1980C (P9), received 13th October 1994

The authors are with the Department of Electrical Engineering, National Taiwan University, Taipei, Taiwan, Republic of China

IEE Proc.-Gener. Transm. Distrib., Vol. 142, No. 4, July 1995
$i_{B} \quad=$ compensator current

$e_{B} \quad=$ compensator voltage

$i_{T} \quad=$ transmission line current

subscripts

$d, q \quad=d$-axjs and $q$-axis stator quantities

$F \quad=$ field circuit

$D, Q, S \quad=$ damper windings

$H P=$ high pressure turbine

$I P \quad=$ medium pressure turbine

$L P A, L P B \quad=$ low pressure turbine

$E X \quad=$ exciter

prefix

$\Delta \quad=$ linearised quantities

\section{Introduction}

Series capacitors have been placed on transmission lines to enhance transmission capability by partially compensating line reactance. These series capacitors, coupled with line reactance, will introduce an oscillation mode which may interact with one of the torsional modes inherent in the mass spring system, resulting in subsynchronous resonance (SSR) [1-3]. To prevent generator shaft damages caused by undamped subsynchronous oscillations, various countermeasures to the SSR problem such as excitation controllers [4-6] and thyristor controlled static VAR compensators [7-9] have been proposed. In a previous work conducted by the authors [9], a proportional integral derivative (PID) static VAR controller was designed to improve the damping characteristics of the SSR modes. It is concluded from the results that subsynchronous oscillations could be effectively damped by the proposed PID static VAR controller. The controller gain was designed based on a nominal operating condition and remained fixed no matter how the generator operating conditions changed. In daily operations, the operating point of a generator will drift over a wide range owing to random load changes or unpredictable disturbances such as a fault in the system. To keep the same damping effect under different loading conditions, it is desirable to adapt the controllers gains in real time. In the literature, adaptive controllers or self-tuning controllers for both SVC and PSS have been proposed for the

Financial support given to this work by the National Science Council of ROC under contract number NSC82-0404-E002-72 is appreciated. 
damping of low frequency electromechanical modes [1012]. In these self-tuning controllers, the system parameters are identified using on-line measurements and a proper set of controller gains are then determined in realtime based on the identified system model. A major disadvantage with this approach is that system identification and gain determination require much computational effort in real time. To overcome this difficulty, artificial neural networks (ANNs) [13], which have found several interesting applications in power systems $[14,15]$, have been employed to tune the parameters of power system stabilisers without the laborious work of system identification [16]. In a recent work [17], the eigenvalues for the SSR modes were estimated by the artificial neura network approach. In general, an artificial neural network is a highly distributed, parallel computation machine which can be used to perform complicated nonlinear mappings between its inputs and outputs in a very short period. Therefore, we propose to adapt the controller gains of the static VAR compensator and excitation controller in real-time by using the artificial neural network in this work. To demonstrate the effectiveness of the proposed ANN based adaptive control scheme, various controllers, including adaptive SVC, adaptive EC, fixed gain SVC, fixed gain EC, optimal SVC, and optimal EC, are designed for the IEEE first benchmark model [18], and then, time domain simulations are performed for systems equipped with these control schemes individually, for the purpose of comparison. It is concluded from the results in this work that the unstable SSR modes can be effectively damped by the proposed adaptive controller over a wide range of operating conditions.

\section{System model}

The IEEE first benchmark model with series capacitor compensated transmission line as shown in Fig. 1 is

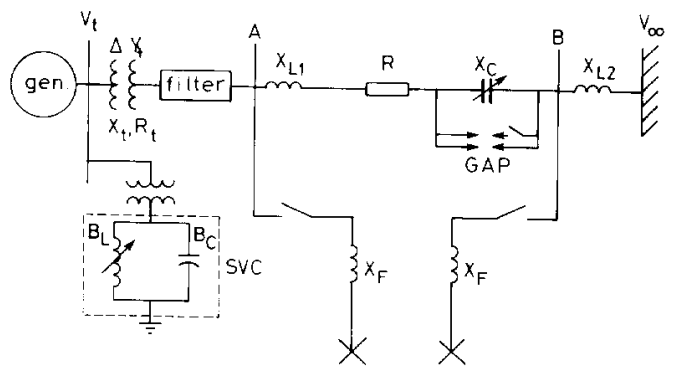

Fig. 1 IEEE first benchmark model with static VAR compensator

employed as the test system of this work. To provide damping effect for the SSR modes on this system, the SVC and EC are utilised as countermeasures. The static VAR compensator comprising a fixed capacitor and a variable inductor is connected through a step-down transformer to the generator bus. The block diagrams of the mass spring system and speed governor system is shown in Fig. 2.

Fig. 3 depicts the block diagram of the static VAR compensator with its controller. Note that the susceptance of the inductor $B_{L}$ can be regulated by adjusting the firing angles of thyristors $\mathrm{TH} 1$ and $\mathrm{TH} 2$ according to the variations in terminal voltage $V_{t}$. The output signal $U$ of the controller is shown in this Figure. The block dia- grams of the static excitation system and the adaptive excitation controller are shown in Fig. 4.

Basically, both the SVC controller and excitation controller are PID controllers with generator speed deviation $\Delta \omega$ and current deviation $\Delta I$ as their inputs. To
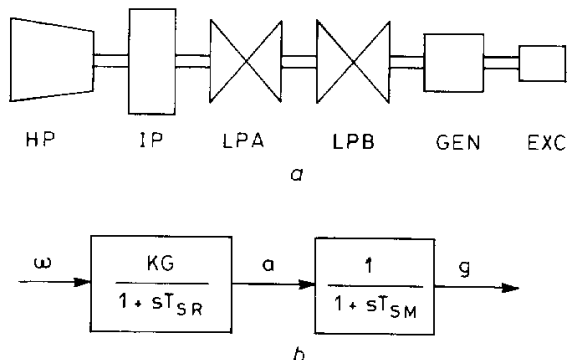

Fig. 2 Block diagrams of mass spring system and speed governor system

a Mass spring system

$b$ governor

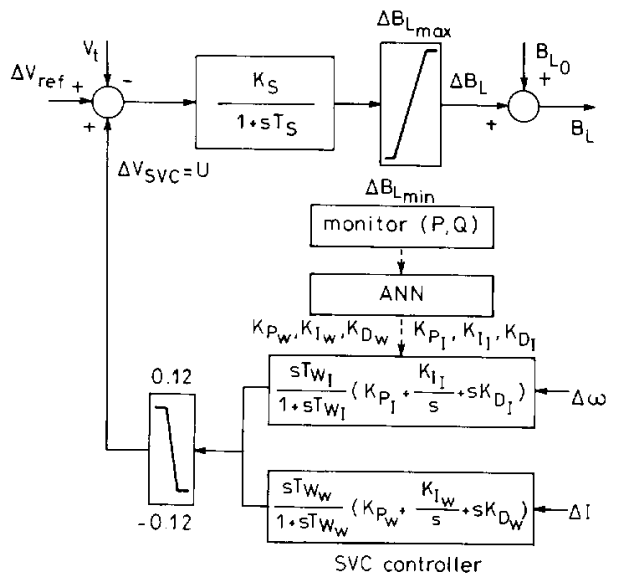

Fig. 3 Block diagram of static VAR compensator with SVC controller

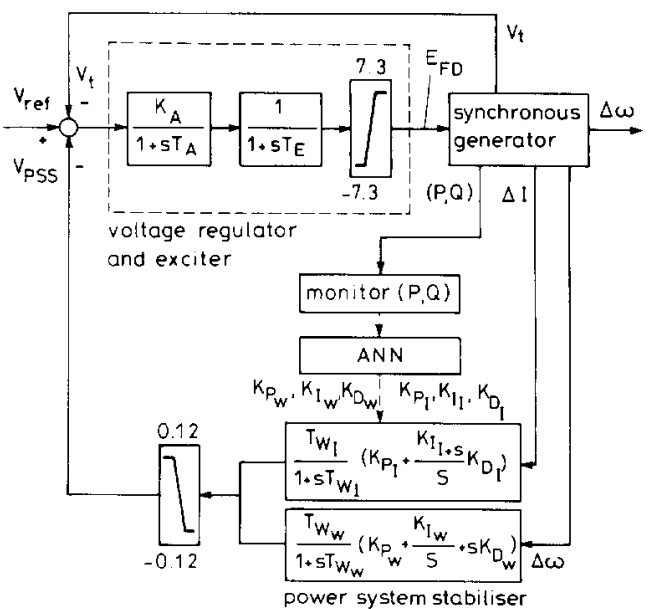

Fig. 4 Block diagram of static excitation system and adaptive excita tion controller

IEE Proc-Gener. Transm. Distrib., Vol. 142, No. 4, July 1995 
keep good damping effects under disturbance conditions, the controller gains $K_{P_{t}}, K_{I_{l}}, K_{D_{t}}, K_{P_{m}}, K_{I_{n}}$, and $K_{D_{m}}$, which are fixed at certain values all the time in a fixed. gain controller, are adapted in real-time by the ANN based on on-line measured generator operating conditions.

In this work, the generator is represented by $D-Q$ axis equivalent circuits $[1,2]$. The model and parameters of the SVC are taken from Reference 4. All system data used in this paper are listed in Appendix 9.1.

\section{Design of a fixed gain controller}

3.1 SVC controller design

In the design of a PID SVC controller, the nonlinear system equations of the study system are linearised around a nominal operating condition first, and the pole placement method in modal control theory [19] is then applied to determine a proper set of controller parameters $T_{W_{\varepsilon}}, T_{W_{\sigma}}, K_{P_{1}}, K_{D_{1}}, K_{P_{\varpi}}, K_{I_{\sigma}}$, and $K_{D_{\varpi}}$, such that all SSR modes can be effectively damped. The linearised nonlinear equations are of the following form:

$$
\begin{aligned}
\dot{X} & =A X(t)+B U(t) \\
Y & =C X(t)
\end{aligned}
$$

where

$$
\begin{aligned}
X= & {\left[\varpi_{H}, \theta_{H}, \varpi_{I}, \theta_{I}, \varpi_{A}, \theta_{A}, \varpi_{B}, \theta_{B}, \varpi, \delta, \varpi_{X},\right.} \\
& \theta_{X}, a, g, T_{H}, T_{A}, T_{i}, e_{c d}, e_{c q}, e_{B d}, e_{B q}, E_{f d}, \\
& \left.i_{\dot{d}}, i_{F}, i_{D}, i_{q}, i_{Q}, i_{S}, i_{T d}, i_{T q}, i_{B d}, i_{B q}, B_{L}, V_{R}\right]^{T}
\end{aligned}
$$

is the state vector, $U(t)$ is the input vector and

$$
Y=[\Delta \omega \Delta I]^{T}
$$

is the output vector.

Then, the eigenvalues of the system without controller can be computed and are summarised in the second column of Table 1 .

From the second column of Table 1, mode 3 is negatively damped while mode 4 is poorly damped. To improve the dampings of the SSR modes, an effective controller is needed.
The PID SVC controller used in this work is a singleinput multiple-output (SIMO) one. Eight unknown variables including two washout time constants $T_{W_{0}}$ and $T_{W_{I}}$ and six gains $K_{P_{\varphi}}, K_{I_{p}}, K_{D_{\sigma}}, K_{P_{I}}, K_{I_{I}}$, and $K_{D_{I}}$, must be determined in the design process. The two washout time constants are determined in advance. They can not be determined through pole placement because a set of improperly assigned poles may lead to negative time constants which are not acceptable. The values of $T_{W_{m}}$, and $T_{W}$ are selected to be 0.5 and $0.05 \mathrm{~s}$, respectively, by a trial and error approach. The objective used in selecting the time constants is to expand the stable operating region as much as possible while maintaining the capability of isolating random noises.

To evaluate the remaining six gains, six prespecified eigenvalues are needed when using the pole placement approach. To do this, the root loci of the system without any controller are first depicted in Fig. 5. An observation
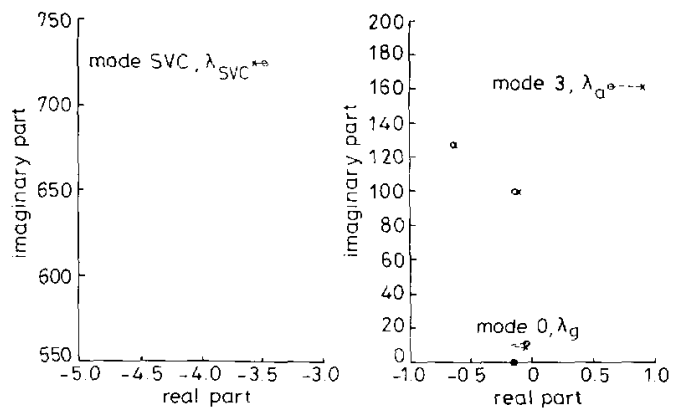

Fig. 5 Root loci of the open-loop system

of the root loci reveals that the modes which are most sensitive to the drifts in loading conditions are the two torsional modes $\lambda_{g}$ and $\lambda_{a}$ and SVC mode. As a result, we can prespecify the six eigenvalues for the three modes as follows:

$$
\begin{aligned}
& -3.0 \pm j 7.5 \\
& -1.6 \pm j 159.27 \\
& -4.5 \pm j 730
\end{aligned}
$$

Table 1 : System eigenvalues at $P=0.9, Q=0.435, V=1.05, X_{c} / X_{t}=0.5$

\begin{tabular}{lccc}
\hline & Open-loop & With fixed gain SVC & With fixed gain EC \\
\hline mode 5 & $-0.182 \pm j 298.177$ & $-0.182 \pm j 298.177$ & $-0.182 \pm j 298.177$ \\
mode 4 & $-0.026 \pm j 202.695$ & $-0.542 \pm j 203.146$ & $-0.289 \pm j 203.03$ \\
mode 3 & $0.765 \pm j 160.691$ & $-1.600 \pm j 159.270$ & $-1.7568 \pm j 159.89$ \\
mode 2 & $-0.642 \pm j 127.065$ & $-0.709 \pm j 126.946$ & $-0.7486 \pm j 126.875$ \\
mode 1 & $-0.110 \pm j 99.430$ & $-0.651 \pm j 98.800$ & $-1.3 \pm j 97$ \\
mode 0 & $-0.155 \pm j 9.722$ & $-3.000 \pm j 5.500$ & $-2.50 \pm j 7$ \\
stator and & $-6.964 \pm j 590.597$ & $-6.415 \pm j 590.372$ & $-7.039 \pm j 590.9481$ \\
network & $-6.984 \pm j 161.833$ & $-4.821 \pm j 163.924$ & $-5.00 \pm j 162.7$ \\
exciter & -499.933 & -499.933 & -500.697 \\
regulator & -101.578 & -101.585 & -133.598 \\
& $-3.462 \pm j 1485.460$ & $-0.768 \pm j 1480.2$ & \\
& $-3.533 \pm j 724.704$ & $-4.5 \pm j 730$ & \\
& $-0.230 \pm j 376.991$ & $-0.238 \pm j 376.988$ & -1.481 \\
& -31.595 & -33.073 & -24.802 \\
& -24.783 & -24.839 & $-26.4856 \pm j 31.8666$ \\
& -20.000 & -19.792 & -15.508 \\
& -10.293 & -3.937 & -4.758 \\
& -6.000 & -6.149 & -3.136 \\
& $-4.684 \pm j 0.46$ & -5.390 & -4.505 \\
& -0.142 & $-3.142 \pm j 0.407$ & -0.138 \\
\hline & -4.477 & -4.820 & \\
& -1.705 & -1.200 & \\
\hline
\end{tabular}


The controller gains can be determined as

$$
\begin{aligned}
& K_{P_{\mathrm{m}}}=72.293 \\
& K_{I_{\mathrm{m}}}=-26.48 \\
& K_{D_{\mathrm{m}}}=1.93428 \\
& K_{P_{I}}=0.155 \\
& K_{I_{I}}=3.835 \\
& K_{D_{I}}=-0.000453
\end{aligned}
$$

under the operating condition of $P=0.9$ and $Q=0.435$.

Column 3 of Table 1 lists the eigenvalues for the system equipped with the SVC and its controller. It is observed that the torsional oscillations are suppressed satisfactorily.

\subsection{Excitation controller design}

Using the same procedures as mentioned above, the parameters of a PID EC can be determined to improve the damping of SSR modes.

The assigned poles are as follows:

$$
\begin{aligned}
& -2.5 \pm j 5.0 \\
& -1.2 \pm j 97.1 \\
& -1.76 \pm j 163.3
\end{aligned}
$$

The controller gains are

$$
\begin{aligned}
& T_{W_{\varpi}}=0.02 \mathrm{~s} \\
& K_{P_{\text {च }}}=486.171 \\
& K_{I_{\text {m }}}=-331.453 \\
& K_{D_{\text {m }}}=3.2142 \\
& T_{W_{I}}=0.23 \mathrm{~s} \\
& K_{P_{I}}=2.39 \\
& K_{I t}=10.876 \\
& K_{D_{I}}=-0.004
\end{aligned}
$$

The eigenvalues of the system with this excitation controller are listed in Column 4 of Table 1.

\section{Application of the ANN to adapt controller gains}

4.1 Artificial neural network

The multilayer feedforward artificial neural network as shown in Fig. 6 will be used in this work to adapt controller gains of both the PID SVC and PID EC in real time. Before the ANN can be used to adapt the controller gains in real time, it is necessary to determine a proper set of values for the connection weights $W_{i j}$. The process of reaching the connection weights is normally carried out off-line and is usually referred to as the training process. In the training process, we first compile a set of training patterns and store these training patterns in the training set. Each training pattern comprises a set of input data and the corresponding output data. In the present work, generator real power output $P$ and reactive power output $Q$, which can be used to characterise generator loading conditions, are selected as the inputs to the ANN. For a generator which is operated at the specified values of $P$ and $Q$, we can proceed to determine a set of PID controller gains $\left(K_{P_{v}}, K_{I_{g}}, K_{D_{m}}, K_{P_{t}}, K_{I}\right.$, and $\left.K_{D_{t}}\right)$ using the pole placement method, and the results are treated as the ANN's output.

\subsection{Application of the ANN to adapt PID controller} gains

As mentioned in the previous Section, for the system equipped with SVC, the three modes $\lambda_{g}, \lambda_{a}$ and $\lambda_{s v c}$ are most sensitive to the drifts in operating points. Therefore,

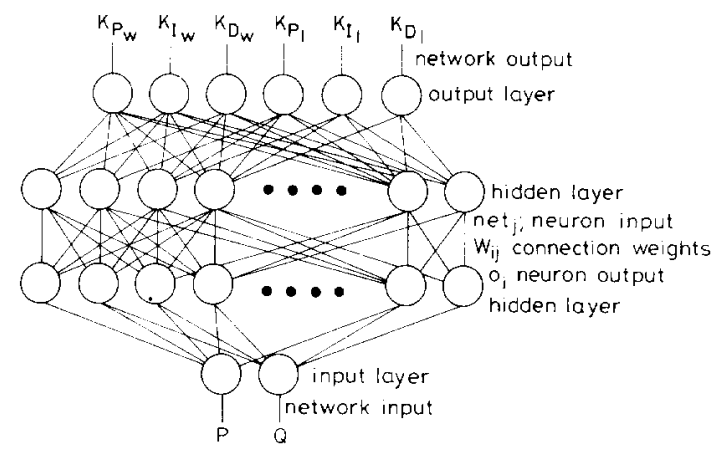

Fig. 6 Multilayer feedforward artificial neural network

on creating training patterns for training the ANN used on tuning SVC controller, these three modes are reassigned as follows:

$$
\begin{aligned}
& -3.0 \pm j 7.5 \\
& -1.6 \pm j 159.27 \\
& -4.5 \pm j 730
\end{aligned}
$$

On the other hand, for the system with only excitation controller, the three modes $\lambda_{g}, \lambda_{\text {stator }}$ and $\lambda_{B}$ are most sensitive to the drifts in operating points. The assigned poles are now selected as:

$$
\begin{aligned}
& -2.5 \pm j 7.0 \\
& -1.3 \pm j 97.0 \\
& -5.0 \pm j 162.7
\end{aligned}
$$

After assigned poles of these two systems have been determined, the following process can be employed for training pattern creation and neural network training for both controllers.

For every possible conbination of $P$ and $Q$ within the interesting range of $(0.6 \leqslant P \leqslant 1.5,-0.4 \leqslant Q \leqslant 1.0)$, we can compute a set of desired controller gains. Each generator operating point and the corresponding PID controller gains constitute a training pattern. These training patterns are stored in the training pattern set for the purpose of determining the connection weights.

A training pattern set comprising 450 training patterns, which cover a wide range of operating conditions, is finally used to train the desired ANN. It should be noted that we use two hidden layers with ten hidden nodes per layer in the present work. The momentum constant $\alpha$ is fixed at 0.9 and an adaptive learning rate as described in Reference 17 is employed.

\section{Optimal control schemes}

The optimal linear state feedback technique is well documented in the literature $[2,20]$. The technique is also applied to design the controllers for both SVC and PSS in this work for the purpose of comparison.

IEE Proc.-Gener. Transm. Distrib., Vol. 142, No. 4, July 1995 
For the linear system (eqn. 1), the control $u(t)$ which minimises the performance index

$$
J=\frac{1}{2} \int_{0}^{\infty}\left(x^{T} Q x+u^{T} R u\right) d t
$$

is given by the feedback control law in terms of system states as

$$
u(t)=-R^{-1} B^{T} S(t) X(t)
$$

where $S(t)$ is the solution of the matrix Riccati equation

$$
0=A^{T} S(t)+S(t) A-S(t) B R^{-1} B^{T} S(t)
$$

The set of nonlinear eqns. 6 can be solved for $S(t)$ in closed form.

It should be noted that the matrix $Q$ is in diagonal form in this study and its elements corresponding to the states of the test system itself are the same in both excitation controller design and SVC controller design.

\section{Simulation results}

To demonstrate the effectiveness of the proposed adaptive controller, time domain simulations are performed on the IEEE first benchmark model as described in Section 2. A $0.1 \mathrm{pu}$ step input in mechanical torque $\Delta T_{m}$ lasting for four cycles is employed as the disturbance. Fig. 7
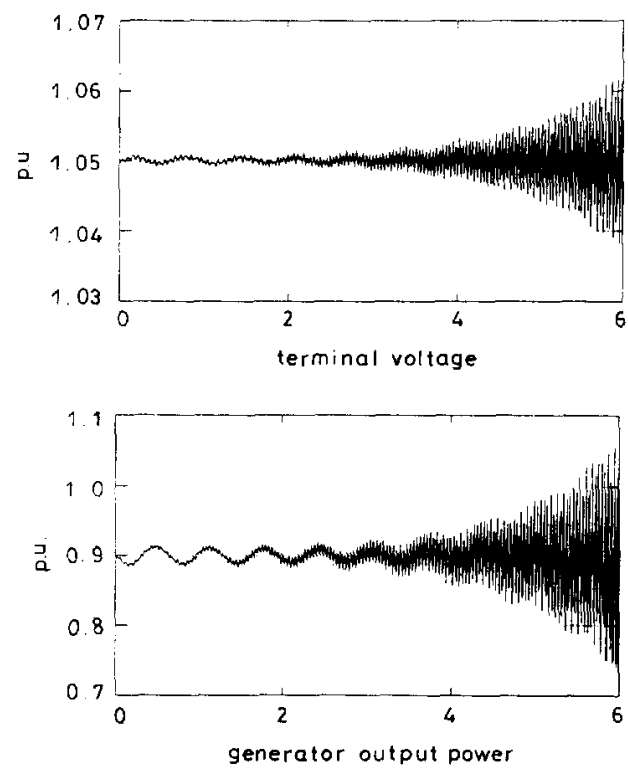

Fig. 7 Dynamic responses of generat or without any controller $P=0.9$, $Q=0.435$ illustrates the response curves of the system without controller under the operating condition considered in Section 3. The system is unstable, just as we predicted in Section 3 based on the negatively damped eigenvalues in the second column of Table 1.

Three different design techniques explained previously are applied to design both the excitation controller and the controller of static VAR compensator. Time domain simulations of the test system equipped with six different control strategies are performed on three loading conditions of generator, i.e. $P=0.9, p f=0.9$ lagging (OPT1), $P=0.7, p f=0.75$ lagging (OPT2), and $P=1.2, p f=0.75$ lagging (OPT3), for comparing the damping effect of each control scheme. The test results are summarised in Table 2.

At OPT1 (Fig. 8), since all controllers are designed at this operating condition, it is observed that all control schemes provide good dampings for the SSR modes at this operating point.

At OPT2 the generator is working at a light loading condition. From Fig. 9, the Optimal SVC cannot maintain stable system operation, but the fixed gain SVC and adaptive gain SVC still work well. As for the excitation control, the optimal PSS have the best damping effect on SSR modes, but its voltage deviation is also the largest. Besides, the ANN tuned adaptive excitation controller can provide much better damping for SSR modes than the fixed gain excitation controller.

At OPT3 (Fig, 10) the generator is working for a heavy load. Both the system with an optimal SVC and the system with a fixed gain PID SVC are unstable, while the ANN tuned adaptive SVC can keep the system in stable operation. But, for excitation controllers, the response characteristic is similar to that at OPT2, but the voltage deviation of the optimal excitation controller is larger.

7 Conclusions

A novel tuning technique based on ANN is proposed to adapt the PID controller gains of both SVC and PSS to control the subsynchronous resonances on capacitor compensated transmission lines. The ANN is trained with a set of training patterns in the training set. Each training pattern includes two portions; one is the inputs of the ANN which contain the operating condition data of generator ( $P$ and $Q$ ) and the other is the outputs of ANN which contain the desired controller gains under the particular operating point. Generalised delta rule with an adaptive momentum constant is adopted during the training process.

Once trained, the ANN is capable of adapting the PID controller gains in real-time based on on-line measured system operating condition. From the time simulation results, it is concluded that, with the controller gains adapted by the ANN, the static VAR compensator can

Table 2: Summary of test results

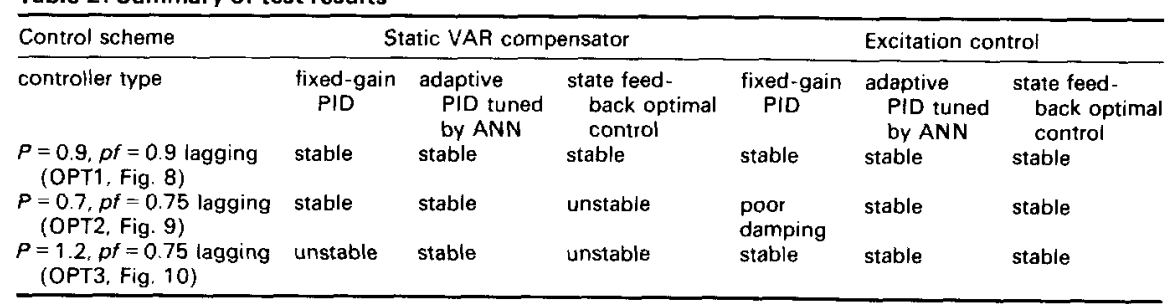



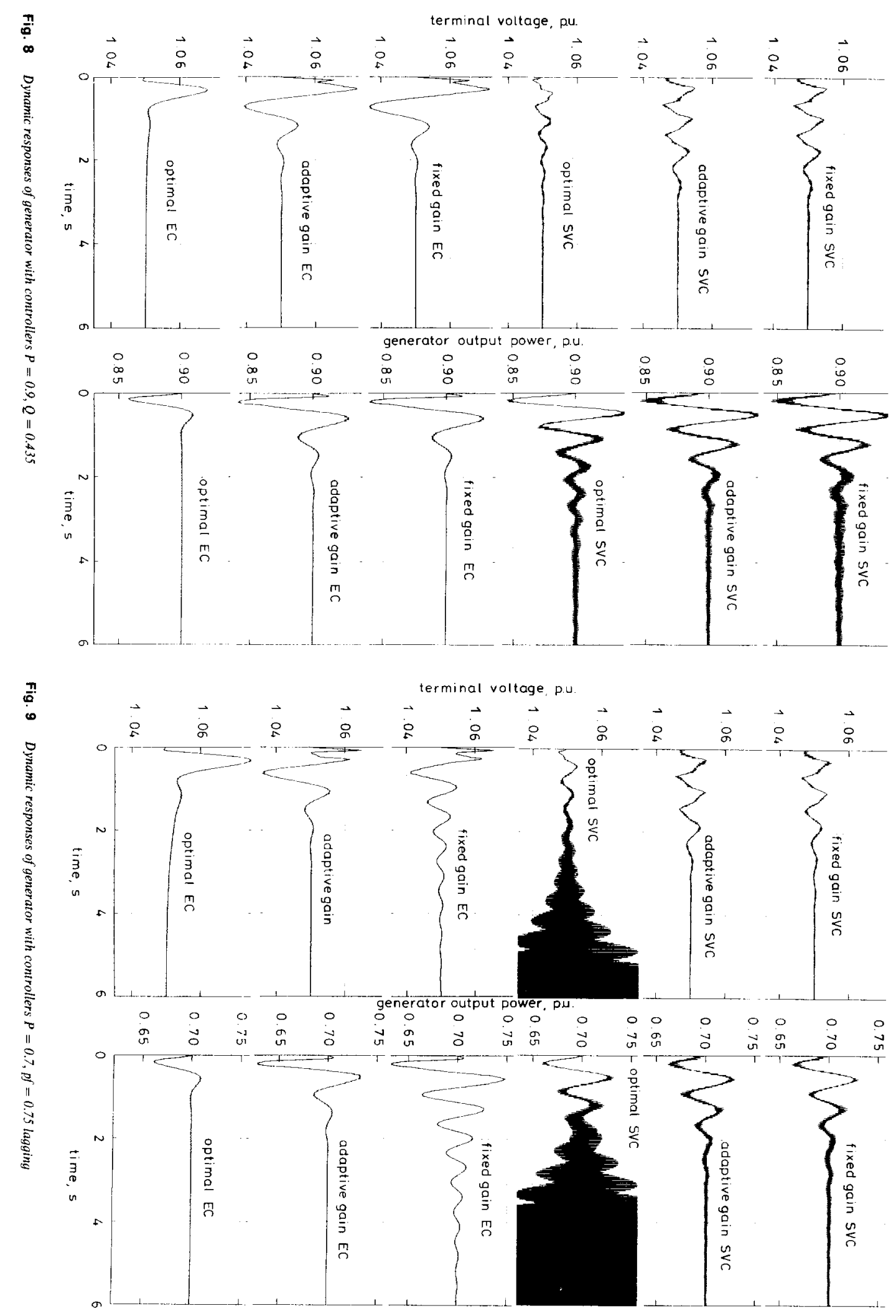
provide better damping characteristics for subsynchronous oscillations over a wide range of operating conditions than both the fixed gain controller and the optimal controller. While both the classical fixed gain controller and the optimal controller may fail in some
It takes a very short period for the $A N N$ to reach the desired controller gains based on the measured $P$ and $Q$. This makes the ANN approach very attractive for the purpose of gain adaptation. Although the training process takes time, this will not cause any disadvantage
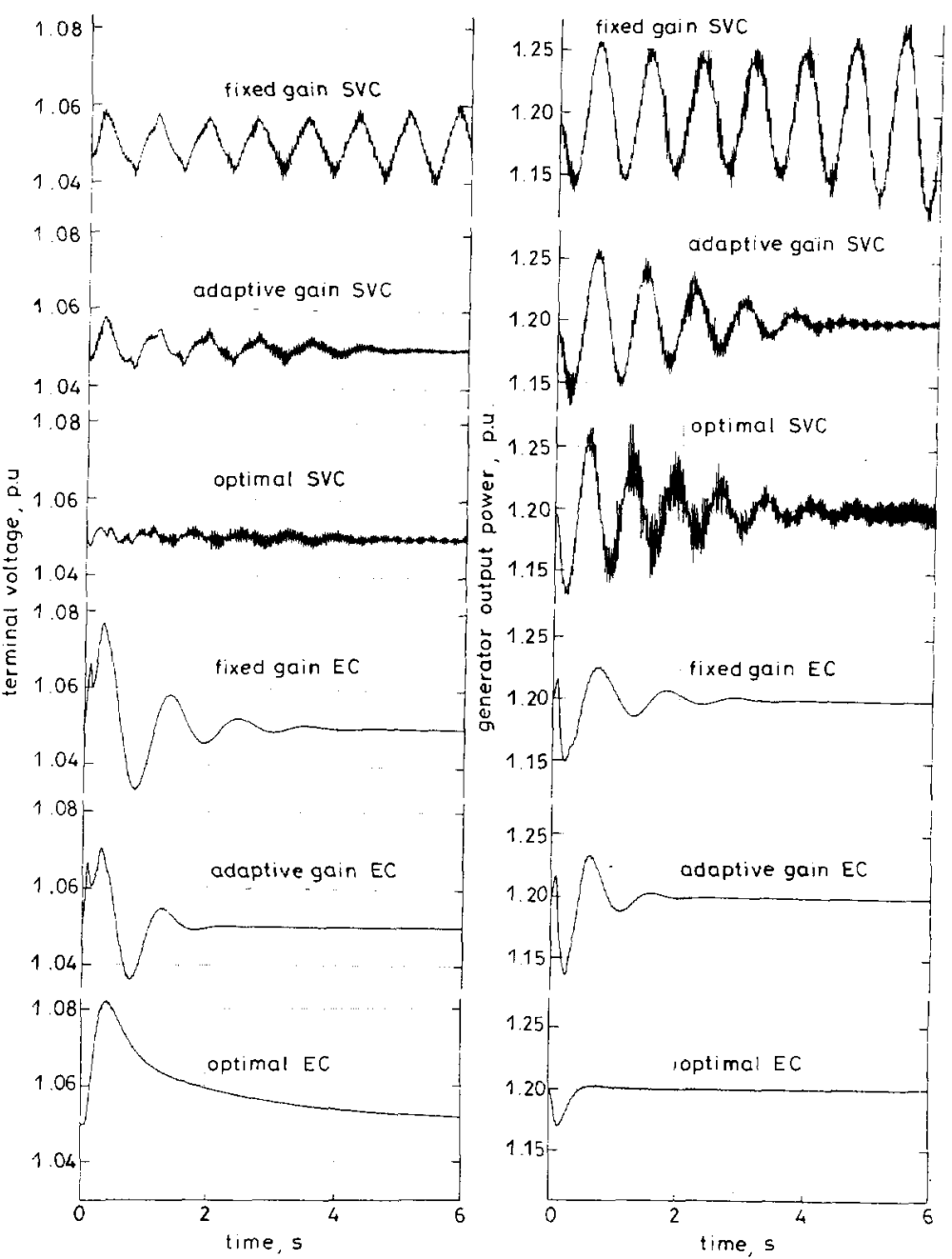

Fig. 10 Dynamic responses of generator with controllers $P=1.2, p f=0.75$ lagging

extreme cases, the adaptive controller still gives satisfactory responses under these conditions.

As for the excitation controllers, the optimal controller has the best damping effect on SSR modes but its voltage deviation is also the largest. The ANN tuned adaptive excitation controller can provide better damping for SSR modes than the fixed gain excitation controller in all cases.

No matter what control technique is employed, the excitation control gives better damping effect for power deviation than the SVC. But, the excitaton controller introduces much larger voltage deviations than the SVC

When considering the damping effect for both generator terminal voltage and power, the ANN tuned adaptive controller is better than optimal controller and fixed gain controller.

IEE Proc.-Gener. Transm. Distrib., Vol. 142, No. 4, July 1995 in applying the ANN since training is usually done offline.

\section{References}

1 ANDERSON, P.M., AGRAWAL, B.L., and VAN NESS, J.E.: 'Subsynchronous resonance in power systems' (IEEE Press, New York, 1990)

2 YU, Y.N.: 'Electric power system dynamics' (Academic Press, New York, 1983)

3 IEEE SSR Working Group: 'Countermeasures to subsynchronous resonance problems', IEEE Trans., 1980, PAS-99, pp. 1810-1818

4 WANG, L., and HSU, Y.Y.: 'Damping of subsynchronous resonance using excitation controllers and static VAR compensators: a comparative study', IEEE Trans., 1988, EC-3, pp. 6-13

5 SARTAWI, M.M., and OOI, B.T.: 'Field excitaton suppression of subsynchronous resonance by real and reactive power signal feedback'. Presented at the IEEE/PES 1978 winter meeting, New York paper A78 293-3 
6 EL-SERAFI, A.M., and SHALTOUT, A.A.: 'Control of subsynchronous resonance oscillations by multi-loop excitation controller'. Presented at the IEEE/PES 1979 winter meeting, paper A79 076-1

7 HAMMAD, A.E., and EL-SADEK, M.: 'Application of a thyristor controlled VAR compensator for damping subsynchronous ascillations in power systems', IEEE Trans., 1984, PAS-103, pp. 198-212

8 BRUCOLI, M., TORELLI, F, and TROVATO, M.: 'A decentral ized control strategy for dynamic shun VAR compensation in interconnected power systems', IEE Proc. C. 1985, 132, pp. 229-236

9 HSU, Y.Y., and WU, C.J.: 'Design of PID static VAR controllers for the damping of subsynchronous oscillations', IEEE Trans., 1988 EC-3, pp. $210-216$

10 CHENG, C.H., and HSU, Y.Y.: 'Damping of generator oscillation using an adaptive static VAR compensator, IEEE Trans., 1992 PWRS-7, pp. 718-725

11 CHENG, S.J., CHOW, Y.S., MALIK, O.P., and HOPE, G.S.: 'An adaptive synchronous machine stabilizer', IEEE Trans., 1986 PWRS-1, (3), pp. 101-109

12 HSU, Y.Y., and LIOU, K.L.: 'Design of self-tuning PID powe system stabilizers for synchronous generators', IEEE Trans., 1987 EC-2, pp. 343-348

13 RUMELHART, D.E., HINTON, G.E., and WILLIAMS, R.J. 'Learning internal representations by error propagation', in RUMELHART, DE, and MCCLELLAND, J.L. 'Parallel distributed processing', 1986. Vol. I, Chap. 8

14 SOBAJIC, D.J., and PAO, Y.H.: 'Artificial neural-nel based dynamic security assessment for electric power systems', IEEE Trans., 1989, PWRS-4, pp. 220-228

15 SANTOSO, M.I and TAN, O.T. 'Neural-net based real-time control of capacitors installed on distribution systems'. Presented at the IEEE/PES 1989 summer meeting, paper 89SM 768-3 PWRS

16 HSU, Y.Y.. and CHEN, C.R.: 'Tuning of power system stabilizers using an artificial neural network', IEEE Trans., 1991, EC-6, pp. 612-619

17 HSU, Y.Y., and JENG, L.H.: 'Analysis of torsional oscillations using an artificial neural network'. Presented at the IEEE/PES 1992 winter meeting paper 92 WM $003-4 \mathrm{EC}$

18 IEEE Committee Report: 'First benchmark model for computer simulation of subsynchronous resonance', IEEE Trans., 1977, PAS-96, pp. $1565-1572$

19 PORTER, B., and CROSSLEY, R.: 'Modal control theory and applications' (Taylor and Francis, 1972)

20 LEWIS, F.L.: 'Applied optimal control and estimation' (Prentice Hall, 1992

21 KAILATH, T.: 'Linear system' (Prentice-Hall, Englewood Cliffs, NJ, 1980), p. 651

\section{$9 \quad$ Appendix}

9.1 System data

Inertia and stiffness constants, $M: \mathrm{s}, K:$ pu torque/rad

$M_{H}=0.185794 \quad K_{\mathrm{HI}}=19.303$

$M_{l}=0.311178 \quad K_{I A}=34.929$

$$
\begin{array}{ll}
M_{A}=1.717340 & K_{A B}=52.038 \\
M_{B}=1.768430 & K_{B G}=70.858 \\
M_{G}=1.736990 & K_{G X}=2.822 \\
M_{X}=0.068433 &
\end{array}
$$

Turbine torques and governor

$$
\begin{array}{lll}
F_{H}=0.3 & T_{C H}=0.3 \mathrm{~s} & K_{G}=25 \\
F_{I}=0.26 & T_{R H}=7.0 \mathrm{~s} & T_{S R}=0.2 \mathrm{~s} \\
F_{A}=0.22 & T_{C O}=0.2 \mathrm{~s} & T_{S M}=0.3 \mathrm{~s} \\
F_{B}=0.22 & &
\end{array}
$$

Exciter and voltage regulator

$$
\begin{array}{ll}
K_{A}=50 & T_{A}=0.01 \mathrm{~s} \\
E_{F D \text { max }}=7.3 \mathrm{pu} & E_{F D \text { min }}=-7.3 \mathrm{pu} \\
U_{E \text { max }}=0.12 \mathrm{pu} & U_{E \text { min }}=-0.12 \mathrm{pu}
\end{array}
$$$$
T_{E}=0.002 \mathrm{~s}
$$

Synchronous generator, $\mathrm{pu}$

$$
\begin{array}{lll}
X_{d}=1.790 & X_{q}=1.710 & X_{f d}=0.04 \\
X_{Q}=0.006 & X_{D}=0.115 & X_{s}=0.245 \\
X_{a d}=1.660 & X_{a q}=1.580 & R_{a}=0.0015 \\
R_{f d}=0.001 & R_{Q}=0.0037 & R_{D}=0.0053 \\
R_{S}=0.0182 & &
\end{array}
$$

Transformer and transmission line

$$
\begin{array}{lll}
R_{T}=0.01 \mathrm{pu} & X_{T}=0.14 \mathrm{pu} & R_{L}=0.02 \mathrm{pu} \\
X_{L 1}=0.5 \mathrm{pu} & X_{L 2}=0.06 \mathrm{pu} & X_{C} / X_{L}=0.5
\end{array}
$$

Initial operating conditions

$$
P=0.9 \mathrm{pu} \quad V_{t}=1.05 \mathrm{pu} \quad p f=0.9 \text { (lagging) }
$$

Static VAR compensator

$$
\begin{array}{lll}
K_{S}=4.0 & T_{S}=0.2 \mathrm{~s} & B_{C}=0.6 \mathrm{pu} \\
L_{T}=0.08 \mathrm{pu} & B_{L_{\text {max }}}=-0.43 \mathrm{pu} & B_{L_{\text {min }}}=-0.47 \mathrm{pu} \\
B_{L 0}=-0.45 & V_{S_{\text {max }}}=0.12 \mathrm{pu} & V_{S_{\text {min }}}=-0.12 \mathrm{pu}
\end{array}
$$

